\title{
Biases in clinical trials with sequential monitoring
}

\author{
Manjula Schou, lan C Marschner* \\ From Clinical Trials Methodology Conference 2011 \\ Bristol, UK. 4-5 October 2011
}

\section{Objectives}

It is well known that a sequentially monitored clinical trial that stops early for benefit has a crude treatment difference that overestimates the true treatment effect. This has led to extended debate in the literature, with some researchers arguing that early stopping is an important source of bias in meta-analyses of clinical trials. We therefore investigated the implications of excluding studies that stopped early, so-called truncated studies, from estimation of treatment effects.

\section{Methods}

The effect of excluding truncated studies was investigated by examining the statistical properties of sequentially monitored studies conditional on reaching the planned final analysis. Using theory and simulation we studied clinical trials with standard sequential rules for stopping early due to benefit. As well as estimation bias, we studied information bias measured as the difference between standard measures of the statistical information, such as sample size, and the actual information based on the conditional sampling distribution.

\section{Results}

We found exclusion of truncated studies leads to both estimation bias and information bias. Treatment differences are underestimated and information is overestimated. Most importantly, the magnitude of information bias is an increasing function of the magnitude of estimation bias. This has important implications for metaanalyses that typically weight by sample size. In particular, it means that studies with the most biased treatment effect are the most overweighted studies in a meta-analysis. The magnitudes of both estimation and information biases can be practically significant. When all studies were included in meta-analyses, both truncated

Department of Statistics, Macquarie University, Sydney, NSW 2109, Australia and non-truncated, the estimation of treatment effects was unbiased.

\section{Conclusions}

Crude methods of analysis for sequentially monitored studies can lead to underestimation bias if truncated studies are excluded from estimation of treatment effects. Furthermore, information bias resulting from this exclusion leads to a double whammy effect, in which the most biased studies are the most overweighted studies in a meta-analysis. Since exclusion of truncated studies is problematic, we advocate wider reporting of adjusted estimates of treatment effects that take account of any interim monitoring, and recommend that all studies, both truncated and non-truncated, are included in meta-analyses.

Published: 13 December 2011

doi:10.1186/1745-6215-12-S1-A50

Cite this article as: Schou and Marschner: Biases in clinical trials with sequential monitoring. Trials 2011 12(Suppl 1):A50.

Submit your next manuscript to BioMed Central and take full advantage of:

- Convenient online submission

- Thorough peer review

- No space constraints or color figure charges

- Immediate publication on acceptance

- Inclusion in PubMed, CAS, Scopus and Google Scholar

- Research which is freely available for redistribution 